\title{
Best proximity point theorems for generalized contractions in partially ordered metric spaces
}

Jingling Zhang, Yongfu Su* and Qingqing Cheng

"Correspondence:

suyongfu@gmail.com;

suyongfu@tjpu.edu.cn

Department of Mathematics, Tianjin

Polytechnic University, Tianjin,

300387, P.R. China

\begin{abstract}
The purpose of this paper is to obtain four best proximity point theorems for generalized contractions in partially ordered metric spaces. Further, our $P$-operator technique, which changes a non-self mapping to a self-mapping, plays an important role. Some recent results in this area have been improved.

MSC: $47 \mathrm{H} 05 ; 47 \mathrm{H} 09 ; 47 \mathrm{H} 10$

Keywords: generalized contraction; fixed point; best proximity point; weak $P$-monotone property
\end{abstract}

\section{Introduction and preliminaries}

Let $A$ and $B$ be nonempty subsets of a metric space $(X, d)$. An operator $T: A \rightarrow B$ is said to be contractive if there exists $k \in[0,1)$ such that $d(T x, T y) \leq k d(x, y)$ for any $x, y \in A$. The well-known Banach contraction principle is as follows: Let $(X, d)$ be a complete metric space, and let $T: X \rightarrow X$ be a contraction of $X$ into itself. Then $T$ has a unique fixed point in $X$.

In the sequel, we denote by $\Gamma$ the functions $\beta:[0, \infty) \rightarrow[0,1)$ satisfying the following condition:

$$
\beta\left(t_{n}\right) \rightarrow 1 \quad \Rightarrow \quad t_{n} \rightarrow 0
$$

In 1973, Geraghty introduced the Geraghty-contraction and obtained Theorem 1.2 as follows.

Definition 1.1 [1] Let $(X, d)$ be a metric space. A mapping $T: X \rightarrow X$ is said to be a Geraghty-contraction if there exists $\beta \in \Gamma$ such that for any $x, y \in X$,

$$
d(T x, T y) \leq \beta(d(x, y)) d(x, y) .
$$

Theorem 1.2 [1] Let $(X, d)$ be a complete metric space, and let $T: X \rightarrow X$ be an operator. Suppose that there exists $\beta \in \Gamma$ such that for any $x, y \in X$,

$$
d(T x, T y) \leq \beta(d(x, y)) d(x, y) .
$$

Then T has a unique fixed point.

\section{Springer}

(O) 2013 Zhang et al.; licensee Springer. This is an Open Access article distributed under the terms of the Creative Commons Attribution License (http://creativecommons.org/licenses/by/2.0), which permits unrestricted use, distribution, and reproduction in any medium, provided the original work is properly cited. 
Obviously, Theorem 1.2 is an extensive version of the Banach contraction principle. Recently, the generalized contraction principle has been studied by many authors in metric spaces or more generalized metric spaces. Some results have been got in partially ordered metric spaces as follows.

Theorem 1.3 [2] Let $(X, \leq)$ be a partially ordered set, and suppose that there exists a metric $d$ such that $(X, d)$ is a complete metric space. Let $f: X \rightarrow X$ be an increasing mapping such that there exists an element $x_{0} \in X$ with $x_{0} \leq f\left(x_{0}\right)$. Suppose that there exists $\beta \in \Gamma$ such that

$$
d(f(x), f(y)) \leq \beta(d(x, y)) d(x, y), \quad \forall y \geq x
$$

Assume that either $f$ is continuous or $X$ is such that if an increasing sequence $x_{n} \rightarrow x \in X$, then $x_{n} \leq x, \forall n$. Besides, iffor each $x, y \in X$, there exists $z \in X$ which is comparable to $x$ and $y$, then $f$ has a unique fixed point.

Theorem 1.4 [3] Let $(X, \leq)$ be a partially ordered set, and suppose that there exists a metric $d \in X$ such that $(X, d)$ is a complete metric space. Let $f: X \rightarrow X$ be a continuous and nondecreasing mapping such that

$$
d(f(x), f(y)) \leq d(x, y)-\psi(d(x, y)) \text { for } y \geq x,
$$

where $\psi:[0, \infty) \rightarrow[0, \infty)$ is a continuous and nondecreasing function such that $\psi$ is positive in $(0, \infty), \psi(0)=0$ and $\lim _{t \rightarrow \infty} \psi(t)=\infty$. If there exists $x_{0} \in X$ with $x_{0} \leq f\left(x_{0}\right)$, then $f$ has a fixed point.

Definition $1.5[4]$ An altering distance function is a function $\psi:[0, \infty) \rightarrow[0, \infty)$ which satisfies

(i) $\psi$ is continuous and nondecreasing.

(ii) $\psi(t)=0$ if and only if $t=0$.

Theorem 1.6 [4] Let $X$ be a partially ordered set, and suppose that there exists a metric $d$ in $X$ such that $(X, d)$ is a complete metric space. Let $T: X \rightarrow X$ be a continuous and nondecreasing mapping such that

$$
\psi(d(T x, T y)) \leq \phi(d(x, y)), \quad \forall y \geq x
$$

where $\psi$ is an altering distance function and $\phi:[0, \infty) \rightarrow[0, \infty)$ is a continuous function with the condition $\psi(t)>\phi(t)$ for all $t>0$. If there exists $x_{0} \in X$ such that $x_{0} \leq T x_{0}$, then $T$ has a fixed point.

Theorem 1.7 [5] Let $(X, \leq)$ be a partially ordered set, and suppose that there exists a metric $d \in X$ such that $(X, d)$ is a complete metric space. Let $f: X \rightarrow X$ be a continuous and nondecreasing mapping such that

$$
\psi(d(f(x), f(y))) \leq \psi(d(x, y))-\phi(d(x, y)) \quad \text { for } y \geq x
$$


where $\psi$ and $\phi$ are altering distance functions. If there exists $x_{0} \in X$ with $x_{0} \leq f\left(x_{0}\right)$, then $f$ has a fixed point.

In 2012, Caballero et al. introduced a generalized Geraghty-contraction as follows.

Definition 1.8 [6] Let $A, B$ be two nonempty subsets of a metric space $(X, d)$. A mapping $T: A \rightarrow B$ is said to be a Geraghty-contraction if there exists $\beta \in \Gamma$ such that for any $x, y \in A$,

$$
d(T x, T y) \leq \beta(d(x, y)) d(x, y) .
$$

Now we need the following notations and basic facts. Let $A$ and $B$ be two nonempty subsets of a metric space $(X, d)$. We denote by $A_{0}$ and $B_{0}$ the following sets:

$$
\begin{aligned}
& A_{0}=\{x \in A: d(x, y)=d(A, B) \text { for some } y \in B\}, \\
& B_{0}=\{y \in B: d(x, y)=d(A, B) \text { for some } x \in A\},
\end{aligned}
$$

where $d(A, B)=\inf \{d(x, y): x \in A$ and $y \in B\}$.

In [7], the authors give sufficient conditions for when the sets $A_{0}$ and $B_{0}$ are nonempty. In [8], the authors prove that any pair $(A, B)$ of nonempty, closed convex subsets of a uniformly convex Banach space satisfies the $P$-property.

Definition 1.9 [9] Let $(A, B)$ be a pair of nonempty subsets of a metric space $(X, d)$ with $A_{0} \neq \emptyset$. Then the pair $(A, B)$ is said to have the P-property if and only if for any $x_{1}, x_{2} \in A_{0}$ and $y_{1}, y_{2} \in B_{0}$,

$$
\left\{\begin{array}{l}
d\left(x_{1}, y_{1}\right)=d(A, B) \\
d\left(x_{2}, y_{2}\right)=d(A, B)
\end{array} \quad \Rightarrow \quad d\left(x_{1}, x_{2}\right)=d\left(y_{1}, y_{2}\right) .\right.
$$

Let $A, B$ be two nonempty subsets of a complete metric space, and consider a mapping $T: A \rightarrow B$. The best proximity point problem is whether we can find an element $x_{0} \in A$ such that $d\left(x_{0}, T x_{0}\right)=\min \{d(x, T x): x \in A\}$. Since $d(x, T x) \geq d(A, B)$ for any $x \in A$, in fact, the optimal solution to this problem is the one for which the value $d(A, B)$ is attained.

In [6], the authors give a generalization of Theorem 1.2 by considering a non-self mapping, and they get the following theorem.

Theorem 1.10 [6] Let $(A, B)$ be a pair of nonempty closed subsets of a complete metric space $(X, d)$ such that $A_{0}$ is nonempty. Let $T: A \rightarrow B$ be a Geraghty-contraction satisfying $T\left(A_{0}\right) \subseteq B_{0}$. Suppose that the pair $(A, B)$ has the P-property. Then there exists a unique $x^{*}$ in $A$ such that $d\left(x^{*}, T x^{*}\right)=d(A, B)$.

Inspired by [6], the purpose of this paper is to obtain four best proximity point theorems for generalized contractions in partially ordered metric spaces. Further, a series of best proximity point problems can be solved by our $P$-operator technique, which changes a non-self mapping to a self-mapping. Some recent results in this area have been improved. 


\section{Main results}

Before giving our main results, we first introduce the weak $P$-monotone property.

Weak $P$-monotone property Let $(A, B)$ be a pair of nonempty subsets of a partially ordered metric space $(X, d)$ with $A_{0} \neq \emptyset$. Then the pair $(A, B)$ is said to have the weak $P$-monotone property if for any $x_{1}, x_{2} \in A_{0}$ and $y_{1}, y_{2} \in B_{0}$,

$$
\left\{\begin{array}{l}
d\left(x_{1}, y_{1}\right)=d(A, B) \\
d\left(x_{2}, y_{2}\right)=d(A, B)
\end{array} \quad \Rightarrow \quad d\left(x_{1}, x_{2}\right) \leq d\left(y_{1}, y_{2}\right)\right.
$$

furthermore, $y_{1} \geq y_{2}$ implies $x_{1} \geq x_{2}$.

Now we are in a position to give our main results.

Theorem 2.1 Let $(X, \leq)$ be a partially ordered set, and let $(X, d)$ be a complete metric space. Let $(A, B)$ be a pair of nonempty closed subsets of $X$ such that $A_{0} \neq \emptyset$. Let $f: A \rightarrow B$ be an increasing mapping with $f\left(A_{0}\right) \subseteq B_{0}$, and let there exist $\beta \in \Gamma$ such that

$$
d(f(x), f(y)) \leq \beta(d(x, y)) d(x, y), \quad \forall y \geq x .
$$

Assume that either $f$ is continuous or that $\bar{A}_{0}$ is such that if an increasing sequence $x_{n} \rightarrow x \in$ $\bar{A}_{0}$, then $x_{n} \leq x, \forall n$. Suppose that the pair $(A, B)$ has the weak P-monotone property. And for some $x_{0} \in A_{0}$, there exists $\hat{x}_{0} \in B_{0}$ such that $d\left(x_{0}, \hat{x}_{0}\right)=d(A, B)$ and $\hat{x}_{0} \leq f\left(x_{0}\right)$. Besides, iffor each $x, y \in \bar{A}_{0}$, there exists $z \in \bar{A}_{0}$ which is comparable to $x$ and $y$, then there exists an $x^{*}$ in $A$ such that $d\left(x^{*}, f x^{*}\right)=d(A, B)$.

Proof We first prove that $B_{0}$ is closed. Let $\left\{y_{n}\right\} \subseteq B_{0}$ be a sequence such that $y_{n} \rightarrow q \in B$. It follows from the weak $P$-monotone property that

$$
d\left(y_{n}, y_{m}\right) \rightarrow 0 \Rightarrow d\left(x_{n}, x_{m}\right) \rightarrow 0
$$

as $n, m \rightarrow \infty$, where $x_{n}, x_{m} \in A_{0}$ and $d\left(x_{n}, y_{n}\right)=d(A, B), d\left(x_{m}, y_{m}\right)=d(A, B)$. Then $\left\{x_{n}\right\}$ is a Cauchy sequence so that $\left\{x_{n}\right\}$ converges strongly to a point $p \in A$. By the continuity of a metric $d$, we have $d(p, q)=d(A, B)$, that is, $q \in B_{0}$, and hence $B_{0}$ is closed.

Let $\bar{A}_{0}$ be the closure of $A_{0}$, we claim that $f\left(\bar{A}_{0}\right) \subseteq B_{0}$. In fact, if $x \in \bar{A}_{0} \backslash A_{0}$, then there exists a sequence $\left\{x_{n}\right\} \subseteq A_{0}$ such that $x_{n} \rightarrow x$. By the continuity of $f$ and the closeness of $B_{0}$, we have $f x=\lim _{n \rightarrow \infty} f x_{n} \in B_{0}$. That is, $f\left(\bar{A}_{0}\right) \subseteq B_{0}$.

Define an operator $P_{A_{0}}: f\left(\bar{A}_{0}\right) \rightarrow A_{0}$, by $P_{A_{0}} y=\left\{x \in A_{0}: d(x, y)=d(A, B)\right\}$. Since the pair $(A, B)$ has the weak $P$-monotone property and $f$ is increasing, we have

$$
d\left(P_{A_{0}} f x, P_{A_{0}} f y\right) \leq d(f x, f y) \leq \beta(d(x, y)) d(x, y), \quad P_{A_{0}} f y \geq P_{A_{0}} f x,
$$

for any $y \geq x \in \bar{A}_{0}$. Obviously, $P_{A_{0}} f$ is increasing. Let $x_{n}, x \in \bar{A}_{0}, x_{n} \rightarrow x$, when $f$ is continuous, then we have

$$
d\left(P_{A_{0}} f x_{n}, P_{A_{0}} f x\right) \leq d\left(f x_{n}, f x\right) \rightarrow 0 \quad \Rightarrow \quad P_{A_{0}} f x_{n} \quad \Rightarrow \quad P_{A_{0}} f x, \quad \text { as } n \rightarrow \infty
$$


Then $P_{A_{0}} f$ is continuous. When $\bar{A}_{0}$ is such that if an increasing sequence $x_{n} \rightarrow x \in \bar{A}_{0}$, then $x_{n} \leq x(\forall n)$, we need not prove the continuity of $P_{A_{0}} f$. For some $x_{0} \in A_{0}$, there exists $\hat{x}_{0} \in B_{0}$ such that $d\left(x_{0}, \hat{x}_{0}\right)=d(A, B)$ and $\hat{x}_{0} \leq f\left(x_{0}\right)$. That is,

$$
d\left(x_{0}, \hat{x}_{0}\right)=d(A, B), \quad d\left(P_{A_{0}} f x_{0}, f x_{0}\right)=d(A, B), \quad \hat{x}_{0} \leq f\left(x_{0}\right) .
$$

By the weak $P$-monotone property, we have $P_{A_{0}} f x_{0} \geq x_{0}$.

This shows that $P_{A_{0}} f: \bar{A}_{0} \rightarrow \bar{A}_{0}$ is a contraction satisfying all the conditions in Theorem 1.3. Using Theorem 1.3, we can get $P_{A_{0}} f$ has a unique fixed point $x^{*}$. That is, $P_{A_{0}} f x^{*}=x^{*} \in A_{0}$, which implies that

$$
d\left(x^{*}, f x^{*}\right)=d(A, B) \text {. }
$$

Therefore, $x^{*}$ is the unique one in $A_{0}$ such that $d\left(x^{*}, f x^{*}\right)=d(A, B)$.

Theorem 2.2 Let $(X, \leq)$ be a partially ordered set, and let $(X, d)$ be a complete metric space. Let $(A, B)$ be a pair of nonempty closed subsets of $X$ such that $A_{0} \neq \emptyset$. Let $f: A \rightarrow B$ be a continuous and nondecreasing mapping with $f\left(A_{0}\right) \subseteq B_{0}$, and let $f$ satisfy

$$
d(f(x), f(y)) \leq d(x, y)-\psi(d(x, y)) \text { for } y \geq x,
$$

where $\psi:[0, \infty) \rightarrow[0, \infty)$ is a continuous and nondecreasing function such that $\psi$ is positive in $(0, \infty), \psi(0)=0$ and $\lim _{t \rightarrow \infty} \psi(t)=\infty$. Suppose that the pair $(A, B)$ has the weak P-monotone property. If for some $x_{0} \in A_{0}$, there exists $\hat{x}_{0} \in B_{0}$ such that $d\left(x_{0}, \hat{x}_{0}\right)=d(A, B)$ and $\hat{x}_{0} \leq f\left(x_{0}\right)$, then there exists an $x^{\prime \prime}$ in $A$ such that $d\left(x^{\prime \prime}, f x^{\prime \prime}\right)=d(A, B)$.

Proof In Theorem 2.1, we have proved that $B_{0}$ is closed and $f\left(\bar{A}_{0}\right) \subseteq B_{0}$. Now we define an operator $P_{A_{0}}: f\left(\bar{A}_{0}\right) \rightarrow A_{0}$ by $P_{A_{0}} y=\left\{x \in A_{0}: d(x, y)=d(A, B)\right\}$. Since the pair $(A, B)$ has the weak $P$-monotone property, by the definition of $f$, we have

$$
d\left(P_{A_{0}} f x, P_{A_{0}} f y\right) \leq d(f x, f y) \leq d(x, y)-\psi(d(x, y)), \quad P_{A_{0}} f y \geq P_{A_{0}} f x,
$$

for any $y \geq x \in \bar{A}_{0}$. Obviously, $P_{A_{0}} f$ is continuous and nondecreasing. For some $x_{0} \in A_{0}$, there exists $\hat{x}_{0} \in B_{0}$ such that $d\left(x_{0}, \hat{x}_{0}\right)=d(A, B)$ and $\hat{x}_{0} \leq f\left(x_{0}\right)$. That is,

$$
d\left(x_{0}, \hat{x}_{0}\right)=d(A, B), \quad d\left(P_{A_{0}} f x_{0}, f x_{0}\right)=d(A, B), \quad \hat{x}_{0} \leq f\left(x_{0}\right) .
$$

By the weak $P$-monotone property, we have $P_{A_{0}} f x_{0} \geq x_{0}$.

This shows that $P_{A_{0}} f: \bar{A}_{0} \rightarrow \bar{A}_{0}$ is a contraction satisfying all the conditions in Theorem 1.4. Using Theorem 1.4, we can get $P_{A_{0}} f$ has a fixed point $x^{*}$. That is, $P_{A_{0}} f x^{*}=x^{*} \in A_{0}$, which implies that

$$
d\left(x^{*}, f x^{*}\right)=d(A, B) .
$$

Therefore, $x^{*}$ is the one in $A_{0}$ such that $d\left(x^{*}, f x^{*}\right)=d(A, B)$. 
Theorem 2.3 Let $X$ be a partially ordered set, and let $(X, d)$ be a complete metric space. Let $(A, B)$ be a pair of nonempty closed subsets of $X$ such that $A_{0} \neq \emptyset$. Let $T: A \rightarrow B$ be a continuous and nondecreasing mapping with $T\left(A_{0}\right) \subseteq B_{0}$, and let $T$ satisfy

$$
\psi(d(T x, T y)) \leq \phi(d(x, y)), \quad \forall y \geq x,
$$

where $\psi$ is an altering distance function and $\phi:[0, \infty) \rightarrow[0, \infty)$ is a continuous function with the condition $\psi(t)>\phi(t)$ for all $t>0$. Suppose that the pair $(A, B)$ has the weak $P$-monotone property. If for some $x_{0} \in A_{0}$, there exists $y_{0} \in B_{0}$ such that $d\left(x_{0}, y_{0}\right)=d(A, B)$ and $y_{0} \leq T x_{0}$, then there exists an $x^{*}$ in $A$ such that $d\left(x^{*}, T x^{*}\right)=d(A, B)$.

Proof In Theorem 2.1, we have proved that $B_{0}$ is closed and $f\left(\bar{A}_{0}\right) \subseteq B_{0}$. Define an operator $P_{A_{0}}: T\left(\bar{A}_{0}\right) \rightarrow A_{0}$ by $P_{A_{0}} y=\left\{x \in A_{0}: d(x, y)=d(A, B)\right\}$. Since the pair $(A, B)$ has the weak $P$-monotone property and $\psi$ is nondecreasing, by the definition of $T$, we have

$$
\psi\left(d\left(P_{A_{0}} T x, P_{A_{0}} T y\right)\right) \leq \psi(d(T x, T y)) \leq \phi(d(x, y)), \quad P_{A_{0}} T y \geq P_{A_{0}} T x,
$$

for any $y \geq x \in \bar{A}_{0}$. Since

$$
\begin{aligned}
& \phi(d(x, y)) \rightarrow 0 \quad \Leftrightarrow \quad d(x, y) \rightarrow 0 \\
& \quad \Rightarrow \quad \psi\left(d\left(P_{A_{0}} T x, P_{A_{0}} T y\right)\right) \rightarrow 0 \quad \Leftrightarrow \quad d\left(P_{A_{0}} T x, P_{A_{0}} T y\right) \rightarrow 0,
\end{aligned}
$$

this shows that $P_{A_{0}} T$ is continuous and nondecreasing. Because there exist $x_{0} \in A_{0}$ and $y_{0} \in B_{0}$ such that $d\left(x_{0}, y_{0}\right)=d(A, B)$ and $y_{0} \leq T x_{0}$, by the weak $P$-monotone property, we have $x_{0} \leq P_{A_{0}} T x_{0}$.

This shows that $P_{A_{0}} T: \bar{A}_{0} \rightarrow \bar{A}_{0}$ is a contraction from a complete metric subspace $\bar{A}_{0}$ into itself and satisfies all the conditions in Theorem 1.6. Using Theorem 1.6, we can get $P_{A_{0}} T$ has a fixed point $x^{\prime \prime}$. That is, $P_{A_{0}} T x^{\prime \prime}=x^{\prime \prime} \in A_{0}$, which implies that

$$
d\left(x^{*}, T x^{*}\right)=d(A, B) \text {. }
$$

Therefore, $x^{*}$ is the one in $A_{0}$ such that $d\left(x^{*}, T x^{*}\right)=d(A, B)$.

Theorem 2.4 Let $(X, \leq)$ be a partially ordered set, and let $(X, d)$ be a complete metric space. Let $(A, B)$ be a pair of nonempty closed subsets of $X$ such that $A_{0} \neq \emptyset$. Let $T: A \rightarrow B$ be a continuous and nondecreasing mapping with $T\left(A_{0}\right) \subseteq B_{0}$, and let $T$ satisfy

$$
\psi(d(T(x), T(y))) \leq \psi(d(x, y))-\phi(d(x, y)) \quad \text { for } y \geq x
$$

where $\psi$ and $\phi$ are altering distance functions. Suppose that the pair $(A, B)$ has the weak P-monotone property. If for some $x_{0} \in A_{0}$, there exists $y_{0} \in B_{0}$ such that $d\left(x_{0}, y_{0}\right)=d(A, B)$ and $y_{0} \leq T x_{0}$, then there exists an $x^{*}$ in $A$ such that $d\left(x^{*}, T x^{*}\right)=d(A, B)$.

Proof In Theorem 2.1, we have proved that $B_{0}$ is closed and $f\left(\bar{A}_{0}\right) \subseteq B_{0}$. Define an operator $P_{A_{0}}: T\left(\bar{A}_{0}\right) \rightarrow A_{0}$ by $P_{A_{0}} y=\left\{x \in A_{0}: d(x, y)=d(A, B)\right\}$. Since the pair $(A, B)$ has the weak $P$-monotone property and $\psi$ is nondecreasing, by the definition of $T$, we have

$$
\psi\left(d\left(P_{A_{0}} T x, P_{A_{0}} T y\right)\right) \leq \psi(d(T x, T y)) \leq \psi(d(T x, T y))-\phi(d(x, y)), \quad P_{A_{0}} T y \geq P_{A_{0}} T x,
$$


for any $y \geq x \in \bar{A}_{0}$. Since

$$
\begin{aligned}
& d(x, y) \rightarrow 0 \quad \Rightarrow \quad \psi(d(x, y))-\phi(d(x, y)) \rightarrow 0 \\
& \quad \Rightarrow \quad \psi\left(d\left(P_{A_{0}} T x, P_{A_{0}} T y\right)\right) \rightarrow 0 \quad \Leftrightarrow \quad d\left(P_{A_{0}} T x, P_{A_{0}} T y\right) \rightarrow 0 .
\end{aligned}
$$

This shows that $P_{A_{0}} T$ is continuous and nondecreasing. Because there exist $x_{0} \in A_{0}$ and $y_{0} \in B_{0}$ such that $d\left(x_{0}, y_{0}\right)=d(A, B)$ and $y_{0} \leq T x_{0}$, by the weak $P$-monotone property, we have $x_{0} \leq P_{A_{0}} T x_{0}$.

This shows that $P_{A_{0}} T: \bar{A}_{0} \rightarrow \bar{A}_{0}$ is a contraction from a complete metric subspace $\bar{A}_{0}$ into itself and satisfies all the conditions in Theorem 1.7. Using Theorem 1.7, we can get $P_{A_{0}} T$ has a fixed point $x^{*}$. That is, $P_{A_{0}} T x^{*}=x^{*} \in A_{0}$, which implies that

$$
d\left(x^{*}, T x^{*}\right)=d(A, B) \text {. }
$$

Therefore, $x^{*}$ is the one in $A_{0}$ such that $d\left(x^{*}, T x^{*}\right)=d(A, B)$.

\section{Competing interests}

The authors declare that they have no competing interests.

\section{Authors' contributions}

All the authors contributed equally to the writing of the present article. All authors read and approved the final manuscript.

\section{Acknowledgements}

This project is supported by the National Natural Science Foundation of China under grant (11071279).

Received: 17 January 2013 Accepted: 14 March 2013 Published: 4 April 2013

\section{References}

1. Geraghty, M: On contractive mappings. Proc. Am. Math. Soc. 40, 604-608 (1973)

2. Amini-Harandi, A, Emami, $\mathrm{H}$ : A fixed point theorem for contraction type maps in partially ordered metric spaces and application to ordinary differential equations. Nonlinear Anal. 72, 2238-2242 (2010)

3. Harjani, J, Sadarangni, K: Fixed point theorems for weakly contraction mappings in partially ordered sets. Nonlinear Anal. 71, 3403-3410 (2009)

4. Yan, F, Su, Y, et al.: A new contraction mapping principle in partially ordered metric spaces and applications to ordinary differential equations. Fixed Point Theory Appl. (2012). doi:10.1186/1687-1812-2012-152

5. Harjani, J, Sadarangni, K: Generalized contractions in partially ordered metric spaces and applications to ordinary differential equations. Nonlinear Anal. 72, 1188-1197 (2010)

6. Caballero, J, et al.: A best proximity point theorem for Geraghty-contractions. Fixed Point Theory Appl. (2012). doi:10.1186/1687-1812-2012-231

7. Kirk, WA, Reich, S, Veeramani, P: Proximinal retracts and best proximity pair theorems. Numer. Funct. Anal. Optim. 24, 851-862 (2003)

8. Abkar, A, Gabeleh, M: Global optimal solutions of noncyclic mappings in metric spaces. J. Optim. Theory Appl. 153 298-305 (2012)

9. Sankar Raj, V: A best proximity point theorems for weakly contractive nonself mappings. Nonlinear Anal. 74 4804-4808 (2011)

doi:10.1186/1687-1812-2013-83

Cite this article as: Zhang et al.: Best proximity point theorems for generalized contractions in partially ordered metric spaces. Fixed Point Theory and Applications 2013 2013:83. 